\title{
RELACIÓN ENTRE EL DÍA DE COLECCIÓN Y LA RECUPERACIÓN DE EMBRIONES EN ALPACAS SUPEROVULADAS
}

\author{
Relationship Between Day of Collection and Embryo Recovery in \\ Superovulated Alpacas
}

\author{
Miriam Cervantes F. ${ }^{1}$, Wilfredo Huanca L. ${ }^{1,2}$, Mario Gonzales C. ${ }^{3}$, \\ Manuel Palomino C. ${ }^{4}$, Víctor Leyva V. ${ }^{1}$
}

\section{Resumen}

Se estudió la relación entre el día de colección y la recuperación embrionaria en alpacas adultas multíparas. Alpacas con folículos? $7 \mathrm{~mm}$ se sometieron a un protocolo de superovulación, se cubrieron con machos fértiles (día 0 = día de servicio), y los embriones se recuperaron en el día $5(\mathrm{G} 1), 6(\mathrm{G} 2)$ y 7 (G3) post cópula, previa evaluación del número y tamaño de los cuerpos lúteos por ecografía transrectal ovárica. En el Exp. 1 se usaron 14 alpacas, que fueron sacrificadas para recuperar los embriones de los cuernos uterino y del oviducto. En el Exp. 2 se realizó la recuperación embrionaria in vivo a 21 alpacas. El lavado de los cuernos uterinos se hizo con $250 \mathrm{ml}$ de PBS a través de un catéter Foley y en los oviductos se hizo con $20 \mathrm{ml}$ de PBS desde la unión útero tubal hacia la fimbria. El número de embriones recuperados de cuerno uterino así como el porcentaje de recuperación embrionaria fue mayor en G2 y G3 respecto a G1 ( $<<0.05)$, mientras que en el oviducto hubo mayor recuperación en G1. Los embriones recolectados de los cuernos uterinos considerados como buenos y excelentes fue de 81.4\% (35/43) en el Exp. 1 y de $74.5 \%$ (38/51) en el Exp. 2, sin encontrar diferencias significativas entre tratamientos dentro de cada experimento. El estadio de desarrollo embrionario más frecuente en ambos experimentos fue de blastocisto eclosionado $(77.7 \%, 73 / 94)$, sin diferencia estadística entre G2 y G3. Se concluye que los embriones pueden ser recuperadas del oviducto hasta el día 5 y del cuerno uterino desde el día 6 post cópula.

Palabras clave: alpaca, eCG, día de colección, recuperación embrionaria, cuerno uterino, oviducto

\section{Abstract}

The relationship between day of collection and embryo recovery was studied in multiparous adult alpacas. Females with $? 7 \mathrm{~mm}$ follicles were superovulated, mated with fertile males (day $0=$ day of service) and embryos were recovered on day $5(\mathrm{G} 1), 6(\mathrm{G} 2)$

\footnotetext{
${ }^{1}$ Laboratorio de Reproducción Animal, Facultad de Medicina Veterinaria, Universidad Nacional Mayor de San Marcos, Lima

${ }^{2}$ E-mail: whuanca2002@yahoo.com

${ }^{3}$ C.I.P. Quimsachata, Estación Experimental ILLPA-Puno, INIA

${ }^{4}$ Laboratorio de Reproducción Animal y Mejoramiento Genético, EFP Medicina Veterinaria, Universidad Nacional de San Cristóbal de Huamanga, Ayacucho
} 
and 7 (G3) post copula, where the number and size of corpora lutea were echographically evaluated. In Exp. 1, 14 females were slaughtered and embryos were recovered from the uterine horns and the oviduct. In Exp. 2, embryos were recoveredin vivo from 21 females. Each uterine horn was flushed with $250 \mathrm{ml}$ of PBS using a Foley catheter; and oviducts were flushed from the utero-tubal junction to the fimbria using $20 \mathrm{ml}$ of PBS. The number and rate of embryos recovered from the uterine horns was higher in G2 and G3 as compared to G1 ( $\mathrm{p}<0.05)$, while in the oviduct was higher in G1. Collected embryos from uterine horns had the condition of excellent and good in 81.4\% (35/43) in Exp. 1 and 74.5\% (38/51) in Exp. 2, and without statistical differences between treatments in each experiment. The most frequent level of embryo development was hatching blastocyst $(77.7 \%, 73 / 94)$, and without differences between G2 and G3. It was concluded that embryos could be recovered until day 5 after mating in oviducts and from day 6 onwards in the uterine horns.

Key words: alpaca, eCG, collection day, embryo recovery, uterine horn, oviduct

\section{INTRODUCCIÓN}

La transferencia de embriones (TE) como técnica de apoyo a programas de mejoramiento genético ha mostrado ser de gran utilidad en la ganadería lechera (Wiggans, 1991), de allí que su adaptación en alpacas tendría similares beneficios (Adams y Ratto, 2001); sin embargo, estudios empleando diversos esquemas y metodblogías Novoa $e t$ al., 1999; Velásquez y Novoa, 1999; Palomino, 2000; Huanca, 2005a) reportan resultados pobres y variables en el número de embriones recuperados (Cervantes, 2008). Los factores que estarían afectando los resultados podrían circunscribirse a las diferencias en la técnica de superovulación y en el día de recuperación de los embriones.

Así, en estudios de Huanca en alpacas (2005a,b), usando las mismas técnicas de superovulación con eCG y día de colección (día 7 post cópula) empleadas en llamas (Huanca et al., 2004), obtuvo una respuesta ovárica promedio de 7.5 cuerpos lúteos; sin embargo, la recuperación embrionaria fue de solo de 1.4 embriones, similar a lo obtenido por Novoa et al. (1999). Estos resultados hacen suponer que la pérdida de embriones podría ser consecuencia de los estadios avanzados de desarrollo; guardando coherencia con los resultados de Bravo et al. (2004) quienes encontraron estadios más avanzados de desarrollo en alpacas tratadas con eCG a diferencia de alpacas no tratadas. Asimismo, se dispone de reportes sobre la recuperación de embriones a nivel de oviducto al día 3 y 4 post cópula (Aparicio et al., 2003).

En base a estas inferencias, se planteó modificaciones en los esquemas hormonales y en los días de colección, con el propósito de estudiar la relación entre el día de colección y la recuperación de embriones, a fin de obtener un mayor número de embriones por hembra donante para su utilización en los programas de mejoramiento genético en alpacas.

\section{Materiales y Métodos}

\section{Procedimiento Experimental}

El estudio se realizó en el Centro de Investigación y Producción Quimsachata, Estación Experimental ILLPA-INIA, ubicado a 4200 msnm en el distrito de Santa Lucía, Puno. La parte experimental se realizó durante los meses de enero y febrero de 2007 (Experimento 1) y de 2008 (Experimento 2).

Se utilizaron 35 alpacas Huacaya hembras adultas (6 a 8 años de edad), con historia de partos normales y con presencia de un folículo mayor o igual a $7 \mathrm{~mm}$, detectado por ecografía ovárica transrectal. Se usaron ma- 
chos adultos (?4 años de edad) de reconocida fertilidad para el servicio. Todos los animales fueron alimentados en pastura natural.

Se realizó dos fases experimentales en un diseño completamente aleatorio. Las alpacas fueron sometidas a un mismo protocolo de superovulación y determinados días de colección de embriones después de la cópula. La evaluación de la presencia de un folículo preovulatorio ?7 mm que permita iniciar el protocolo de superovulación se hizo con un ecógrafo ALOKA SSD 500 y transductor lineal de 7.5 MHz.

El protocolo de superovulación fue una modificación del protocolo de Huanca (2005a,b). Se administró 650 UI de eCG dos días después de la ovulación inducida con 1 $\mathrm{ml}$ de GnRH im (0.0042 $\mathrm{mg}$ acetato de buserelina), cinco días después se indujo la luteólisis con $1 \mathrm{ml} \mathrm{de} \mathrm{PGF}_{2 \text { ? }}$ im $(0.25 \mathrm{mg}$ cloprostenol), y al día siguiente se evaluó el número y el tamaño de folículos ováricos por ecografía transrectal y se procedió al servicio (>15 min) con macho fértil. En la cópula se controló la correcta penetración del pene del macho. Al final del servicio, las hembras recibieron $1 \mathrm{ml} \mathrm{im}$ de $\mathrm{GnRH}(0.0042 \mathrm{mg}$ acetato de buserelina).

Las alpacas servidas se distribuyeron al azar según día de colección de embriones (día 0 = día de servicio) en tres grupos experimentales: día 5 (G1), 6 (G2) y 7 (G3). Previo a la recuperación embrionaria, se evaluó el número y tamaño de los cuerpos lúteos por ecografía transrectal ovárica.

\section{Experimento 1}

Se emplearon cuatro alpacas por tratamiento. Los animales se sacrificaron el día de la recuperación embrionaria y el tracto reproductivo se removió cuidadosamente. El lavado de los cuernos uterinos se hizo con la técnica de lavado uterino transcervical correspondiente al método de recuperación por circuito abierto con flujo discontinuo descrito en bovinos (Palma, 2008). Se evaluó la cali- dad morfológica y estadio de desarrollo embrionario, tomando como referencia las clasificaciones establecidas por Lehn-Jensen (citado por Callesen et al., 1995). Asimismo, se registró el tamaño de los embriones.

Además, se procedió al lavado de los oviductos para comprobar la presencia de embriones en oviductos entre el día 5 a 7 post cópula. Se diseccionó el mesosalpinx para eliminar la forma tortuosa del oviducto y facilitar el lavado. Se inyectó $10-15 \mathrm{ml}$ de medio de lavado temperado $\left(37^{\circ} \mathrm{C}\right)$ a base de PBS a nivel de la unión útero-tubal, recuperándose el líquido por el extremo opuesto (fimbria) sobre una placa petri estéril grande (previamente rayada).

\section{Experimento 2}

La recuperación embrionaria fue in vivo (método no quirúrgico), utilizando siete animales por tratamiento. El lavado de los cuernos uterinos se hizo en formal similar al Experimento 1. Sin embargo, en este caso, se colocó anestesia epidural a nivel de la base de la cola (lidocaina al 2\%) y se desinfectó la zona vulvar; además, al término del lavado de los cuernos uterinos, las alpacas recibieron $1 \mathrm{ml}$ im de PGF $_{2 \text { ? }}$ (0.25 mg cloprostenol). El proceso de identificación y clasificación de los embriones fue similar al Experimento 1 .

\section{Evaluaciones}

Se evaluó la calidad morfológica y del estadio de desarrollo de los embriones recuperados, el porcentaje de recuperación de embriones (considerando el número de embriones recuperados por lavado uterino transcervical entre el número de cuerpos lúteos detectados ecográficamente el día de la recuperación embrionaria), y el porcentaje de ovulación (considerando el número de cuerpos lúteos detectados el día de la recuperación embrionaria entre el número de folículos superestimulados $? 7 \mathrm{~mm}$ previos a la cópula). Se empleó la diferencia de proporciones para establecer la significancia estadística entre los grupos. 
El número de folículos superestimulados $\geq 7 \mathrm{~mm}$ previo a la cópula, número de cuerpos lúteos y número de embriones recuperados por grupo se evaluó empleando la prueba de Kruskall Wallis. El tamaño de los folículos y cuerpos lúteos se evaluó con la prueba de ANOVA de una vía. El tamaño de los embriones se evaluó con la prueba T Student independiente. Asimismo, se determinó el coeficiente de correlación entre el número promedio de cuerpos lúteos observados y el de embriones recuperados en los grupos en estudio.

Los análisis estadísticos se realizaron con el paquete estadístico Stata 9.2 (StataCorp, Texas, USA), y las diferencias se consideraron significativas cuando $p<0.05$.

\section{Resultados}

El número promedio de embriones recuperados de cuerno uterino, así como el porcentaje de recuperación embrionaria fue significativamente mayor en G2 y G3, respecto a $\mathrm{G} 1(\mathrm{p}<0.05)$, pero sin diferencias estadísticas entre G2 y G3 en ambos experimentos (Cuadro 1). En tres alpacas del G1 (Exp. 2) se hizo un segundo lavado transcervical al día siguiente encontrando más de un embrión. A nivel de oviducto, el número de embriones fue mayor para G1 (Cuadro 1). Se observó una gran variabilidad individual en el número de embriones recuperados, llegándose a obtener hasta 11 embriones en una hembra.

Cuadro 1. Recuperación embrionaria en tractos reproductivos de alpacas luego del sacrificio (Experimento 1, $\mathrm{n}=4,2007$ ) y por método no quirúrgico (Experimento 2, $\mathrm{n}=7$, 2008)

\begin{tabular}{cccccc}
\hline \multirow{2}{*}{$\begin{array}{l}\text { Día de recuperación } \\
\text { de embriones post } \\
\text { cópula }\end{array}$} & $\begin{array}{c}\text { Embriones en } \\
\text { oviducto }\end{array}$ & $\begin{array}{c}\text { Embriones en } \\
\text { cuerno uterino }\end{array}$ & $\begin{array}{c}\text { Relación entre } \\
\text { embriones de } \\
\text { cuernos y CL }\end{array}$ \\
\cline { 2 - 6 } & Prom. \pm d.e. & $\mathrm{n}$ & Prom. \pm d.e. & $\mathrm{n}$ & Prom. \pm d.e. \\
\hline Experimento 1 & $2.3 \pm 0.4^{\mathrm{a}}$ & 9 & $0.2 \pm 0.5^{\mathrm{a}}$ & 1 & $12.5 \pm 25.0^{\mathrm{a}}$ \\
5 & $0.4 \pm 0.5^{\mathrm{a}}$ & 2 & $3.8 \pm 4.0^{\mathrm{b}}$ & 19 & $61.7 \pm 23.1^{\mathrm{b}}$ \\
6 & $0.2 \pm 0.4^{\mathrm{a}}$ & 1 & $4.6 \pm 1.1^{\mathrm{b}}$ & 23 & $42.9 \pm 30.9^{\mathrm{b}}$ \\
7 & & & & & \\
Experimento 1 & & & $0^{\mathrm{a}}$ & 0 & 0 \\
5 & & & $4.3 \pm 2.4^{\mathrm{b}}$ & 30 & $46.8 \pm 21.7^{\mathrm{b}}$ \\
6 & & & $3.0 \pm 2.9^{\mathrm{b}}$ & 21 & $35.7 \pm 27.0^{\mathrm{b}}$ \\
\hline
\end{tabular}

a,b Superíndices diferentes dentro de experimentos y en una misma columna indican diferencia estadística $(p<0.05)$ 
Cuadro 2. Tamaño $(\mathrm{mm})$ de embriones recuperados en tractos reproductivos de alpacas luego del sacrificio (Experimento 1, $\mathrm{n}=4,2007$ ) y por método no quirúrgico (Experimento 2, $\mathrm{n}=7,2008$ )

\begin{tabular}{ccccc}
\hline $\begin{array}{c}\text { Día de recuperación de } \\
\text { embriones post cópula }\end{array}$ & \multicolumn{2}{c}{ Embriones en oviducto } & \multicolumn{2}{c}{ Embriones en cuerno uterino } \\
\hline & $\mathrm{n}$ & Prom. \pm d.e. & $\mathrm{n}$ & Prom. \pm d.e. \\
\hline Experimento 1 & 9 & $0.169 \pm 0.019^{\mathrm{a}}$ & 1 & 0.200 \\
5 & 2 & $0.165 \pm 0.02^{\mathrm{a}}$ & 19 & $0.223 \pm 0.039^{\mathrm{a}}$ \\
6 & 1 & 0.160 & 23 & $0.411 \pm 0.147^{\mathrm{b}}$ \\
7 & & & & \\
Experimento 2 & & & 0 & 0 \\
5 & & & 30 & $0.248 \pm 0.052^{\mathrm{a}}$ \\
6 & & & 21 & $0.470 \pm 0.220^{\mathrm{b}}$ \\
\hline
\end{tabular}

a,b Superíndices diferentes dentro de experimentos y en una misma columna indican diferencia estadística $(p<0.05)$

La calidad morfológica de los embriones recolectados de los cuernos uterinos considerados como buenos y excelentes fue del $81.4 \%$ (35/43) en el Experimento 1 y de $74.5 \%$ (38/51) en el Experimento 2, y del $100 \%$ en embriones recuperados del oviducto (12/12), sin encontrar diferencias significativas entre tratamientos dentro de cada experimento. En total, el $80.2 \%$ de los embriones recuperados presentó una calidad de bueno a excelente (85/106).

El estadio de desarrollo embrionario más frecuente en ambos experimentos fue de blastocisto eclosionado (77.7\%, 73/94), no encontrándose diferencia entre los grupos G2 y G3, mientras que en G1 se recuperó un solo embrión. La frecuencia de blastocistos eclosionados en el Experimento 1 fue de $72.1 \%$ (31/43) y en el Experimento 2 fue de $82.4 \%$ (42/51). A nivel de oviducto, de los 12 embriones recuperados, 9 (75\%) se encon- traban en estadio de mórula compacta. El tamaño de los embriones fue significativamente mayor en el día 7 (G3) que en el día 6 (G2) post cópula en ambos experimentos $(\mathrm{p}<0.05)$.

Se determinó que el número de folículos detectados previo a la monta y el número de cuerpos lúteos detectados el día de la recuperación embrionaria estaban moderadamente correlacionados $(\mathrm{r}=0.7, \mathrm{p}<0.05)$; mientras que el número de cuerpos lúteos detectados y el número de embriones recuperados de útero tuvo una correlación baja $(\mathrm{r}=0.4$, $\mathrm{p}<0.05)$.

\section{Discusión}

El número de embriones recuperados de cuerno uterino fue significativamente menor en G1, toda vez que solo se halló un em- 
brión. Sin embargo, se pudo encontrar nueve embriones a nivel de oviducto en comparación al bajo número encontrado en G2 (2) y G3 (1), aunque es posible que una parte del número total de embriones presentes en oviducto se halla perdido debido a fallas en la aplicación de la técnica de recuperación. No obstante, estos resultados indican que los embriones de las alpacas superovuladas del presente estudio estarían pasando del oviducto al útero luego del día 5 post cópula. Aparicio et al. (2003) reportaron la recuperación de embriones a nivel de oviducto al día 3 y 4 post cópula. El lavado transcervical en el día 6 post cópula, que no estuvo programado en el estudio, mostró la presencia de embriones, lo cual estaría demostrando que los embriones aun se encontraban en el oviducto al día 5 post cópula.

La calidad morfológica de los embriones recuperados en los grupos en estudio fue mayoritariamente de buena a excelente, aspecto importante considerando que la evaluación morfológica de la calidad embrionaria puede proveer de valiosa información para predecir las tasas de preñez post-transferencia, debido a que la transferencia de embriones de este tipo de embriones resultan en mayores tasas de preñez respecto a los embriones de mala calidad en vacas (Linder y Wrigth, 1983; Hasler, 2001), y a los de mediana calidad en ovinos (Angela et al., 2006).

El mayor porcentaje de embriones se encontraba en estadio de blastocisto eclosionado, tanto al día 6 como al día 7 post cópula, y solo se encontró un embrión en estadio de mórula al día 5; resultado que difiere al estudio de Bravo et al. (2004), quienes reportan embriones en estadio de blastocisto al día 6 y blastocisto expandido al día 7 . En el caso de vacas, los ovocitos producidos por hembras superovuladas no son liberados simultáneamente, sino dentro de un periodo de varias horas, lo que puede explicar la variación en el estadio de desarrollo de los embriones recuperados; es así que Callensen $e t$ al. (1986, citado por Palma, 2008) reportan que vacas estimuladas empiezan a ovular 24 horas después del pico de LH, pero la ovulación se prolonga aún más allá de las 33 horas, de modo que los ovocitos no son fecundados en el mismo momento. Así también, la variación de la respuesta superovulatoria puede estar asociada con el animal donador (Callesen et al., 1995), incluyendo sus cualidades genéticas (Palma, 2008).

De igual manera, los resultados del presente trabajo indicarían que alpacas tratadas con eCG ocurriría un desarrollo embrionario acelerado respecto a otras especies, al encontrar embriones en estadio de blastocisto eclosionado desde el día 6 post cópula. Los blastocistos de los bovinos eclosionan a los 8 a 9 días post cópula (Palma, 2008) o a los 10 días post estro (Massip et al., 1982). En llamas, McEvoy et al. (1992, citado por Ratto, 2005) refieren que el acelerado rango de desarrollo embrionario en camélidos sudamericanos podría estar relacionado con el temprano reconocimiento maternal de la preñez, el cual fue postulado en base a un descenso temporal de los niveles de progesterona, sugiriendo que los signos del embrión para su reconocimiento deberían ser transmitidos antes del día 10 post monta con el fin de mantener el cuerpo lúteo de la preñez (Aba et al., 1997).

Por otro lado, el hecho que los embriones no presenten zona pelúcida podría indicar una mayor susceptibilidad de dañarse debido a su mayor fragilidad (Palma, 2008). Esto podría haber afectado el número total de embriones recuperados por lavado uterino transcervical en las alpacas superovuladas.

El número de embriones producidos es inherente a cada hembra donante, dependiendo en parte de los folículos que responden a la superovulación; asimismo, se considera que es improbable controlar los mecanismos intraováricos que determinan la dinámica folicular (Moor et al., 1984). No obstante, la variación en el número de embriones recuperados por animal es un aspecto que no debiera ser ignorado (Merton et al., 2003). Se dispone de estudios que señalan la posibili- 
dad de seleccionar hembras donantes en base al número de folículos pequeños al comienzo del tratamiento superovulatorio, dada su estrecha correlación con la respuesta superovulatoria. De igual manera, se ha observado que ovejas con alto número de folículos en la primera onda folicular presentaron una mejor respuesta superovulatoria en términos de cuerpos lúteos y calidad embrionaria (Mossa et al., 2007). Asimismo, se indica que el tipo de respuesta a un primer tratamiento superovulatorio tiende a ser similar en un segundo (Cordeiro et al., 2002), tercero (Lamberson and Lambeth, 1986) e incluso a lo largo de diez tratamientos (Donaldson and Perry, 1983), aunque hay otros trabajos que reportan una baja repetibilidad y heredabilidad (Tonhati et al., 1999).

La baja correlación entre el número de cuerpos lúteos y el número de embriones recuperados difiere de correlaciones en bovinos donde se reportan correlaciones mayores (Kanuya et al., 1997).

\section{Conclusiones}

Los embriones de alpacas superovuladas pueden ser recuperados del oviducto hasta el día 5 , y de los cuernos uterinos desde el día 6 post cópula.

\section{Agradecimientos}

Los autores agradecen al Proyecto PROCOM 2006 "Biotecnologías Reproductivas en el Mejoramiento Genético de Camélidos. Fase II" del CONCYTEC y al Sub Proyecto «Biotecnologías reproductivas como herramientas para la mejora genética de alpacas y llamas» INCAGRO - UNMSM, por el apoyo financiero; además agradecen a los profesionales, personal técnico y estudiantes presentes en el Centro de Investigación Illpa-Quimsachata, Puno, por la asistencia brindada durante la ejecución del trabajo.

\section{Literatura Citada}

1. Aba M, Bravo P, Forsberg M, Kindahl H. 1997. Endocrine changes during early pregnancy in the Alpaca. Anim Reprod Sci 47: 273-279.

2. Adams G, Ratto M. 2001. Reproductive biotechnology in South American camelids. Rev Inv Vet, Perú Supl 1: 134141.

3. Angela $S$, Prefac $G$, Paul $T$, Alexandru S, Dana T. 2006. Evaluation of sheep embryo quality by morphologic methods - advantages and disadvantages. J Tekirdag Agric Faculty 3(1): 68-70.

4. Aparicio M, Leyva V, Novoa C, García W. 2003. Efecto de la copulación durante el celo postovulatorio en la mortalidad embrionaria en alpacas. Rev Inv Vet, Perú 14(1): 24-32.

5. Bravo W, Cosio E, Alarcón V, Ordoñez C. 2004. The first 10 days of the alpaca embryo. En: $15^{\text {th }}$ International Congress on Animal Reproduction. Brazil. p 84.

6. Callesen H, Lovendahl P, Bak A, Greve T. 1995. Factors affecting the developmental stage of embryos recovered on day 7 from superovulated dairy cattle. J Anim Reprod 73: 15391543.

7. Cervantes M. 2008. Momento óptimo post cópula para la recuperación de embriones del útero de alpaca mediante método no quirúrgico. Tesis de Maestría. Lima: Univ. Nacional Mayor de San Marcos. 77 p.

8. Cordeiro MF, Lima-Verde JB, LopesJúnior ES, Teixeira DIA, Farias L N, Salles HO, Simplício AA, Rondina D, Freitas VJF. 2002. Embryo recovery rate in Santa Inês ewes subjected to successive superovulatory treatments with $\mathrm{pFSH}$. Theriogenology 57: 12631272.

9. Donaldson LE, Perry B. 1983. Embryo production by repeated superovulation of commercial donor cows. Theriogenology 20: 163-168. 
10. Hasler JF. 2001. Factors affecting frozen and fresh embryo transfer pregnancy rates in cattle. Theriogenology 56: 1401-1415.

11. Huanca W. 2005a. Aplicación de biotecnologías en el mejoramiento genético de llamas y alpacas. Fase I: Desarrollo y evaluación de un protocolo de transferencia de embriones. CSI. Boletín 55: 8-12.

12. Huanca W. 2005b. Aplicación de biotecnologías reproductivas en especies domésticas y silvestres de camélidos sudamericanos. Agrociencia Vol. IX (12):505-509.

13. Huanca W, Ratto M, Santiani A, Cordero A, Huanca T. 2004. Embryo transfer in camelids: study of a reliable superovulatory treatment in llamas. In: $4^{\text {th }}$ European Symposium on South American Camelids. Germany.

14. Kanuya N, Callesen H, Hyttel P, Assey R, Greve, T. 1997. Superovulatory response of dairy cattle (Bos taurus) in a tropical environment. Theriogenology 47(8): 1583-1593.

15. Lamberson WR, Lambeth VA. 1986. Repeatability of response to superovulation in Brangus cows. Theriogenology 26: 643-648.

16. Linder GM, Wright RW. 1983. Bovine embryo morphology and evaluation. Theriogenology 20: 407-416.

17. Massip A, Mulnard J, Vanderzwalmen P, Hanzen C, Ectors F. 1982. The behaviour of cow blastocyst in vitro: cinematographic and morphometric analysis. J Anat 134: 399-405.

18. Merton JS, de Roos APW, Mullaart E, de RuighL, Kaal L, Vos PL, Dieleman SJ. 2003. Factors affecting oocyte quality and quantity in commercial application of embryo technologies in the cattle breeding industry. Theriogenology 59: 651-674.

19. Moor RM, Kruipp ThAM, Green D. 1984. Intraovarian control of folliculogenesis: Limits to superovulation? Theriogenology 21: 103-116.

20. Mossa F, Duffy P, Naitana S, Lonergan $P$, Evans A. 2007. Association between numbers of ovarian follicles in the first follicle wave and superovulatory response in ewes. Anim Reprod Sci 100: 391-396.

21. Novoa C, Franco E, García W, Pezo D. 1999. Dosis de gonadotropinas (eCG y hCG), superovulación y obtención de embriones en alpacas. Rev Inv Vet 10: 48-53.

22. Palma G. 2008. Biotecnología de la reproducción. $2^{\mathrm{a}}$ ed. Buenos Aires: Ed Producción Gráfica Integral. 669 p.

23. Palomino H. 2000. Biotecnología del transplante y micromanipulación de embriones de bovinos y camélidos de los Andes. Perú: AFA Ed Importadores. 444 p.

24. Ratto M. 2005. Ovarian follicular synchronization, ovulation and oocyte development in llamas and alpacas. Thesis PhD. Canada: University of Saskatchewan. $185 \mathrm{p}$.

25. Tonhati H, Lobo RB, Oliveira HN. 1999. Repeatability and heritability of response to superovulation in holstein cows. Theriogenology 51: 1151-1156.

26. Velásquez C, Novoa C. 1999. Superovulación con PMSG aplicada en fase folicular y fase luteal en alpacas. Rev Inv Vet 10: 39-47.

27. Wiggans G. 1991. National genetic improvement programs for dairy cattle in the United States. J Anim Sci 69: 38533860 . 